\title{
Cosmic microwave background bounds on primordial black holes
}

\section{Pasquale Dario Serpico*}

Laboratoire d'Annecy-le-Vieux de Physique Théorique (LAPTh),

Université Savoie Mont Blanc \& CNRS, 74941 Annecy Cedex, France

E-mail: serpico@lapth.cnrs.fr

We review several cosmic microwave background constraints on primordial black holes, in particular if they constitute a significant fraction of the dark matter in the universe. The importance of these constraints in the light of some interpretations of the recent detection of gravitational waves from massive stellar black hole mergers is emphasized.

The European Physical Society Conference on High Energy Physics

5-12 July, 2017

Venice

${ }^{*}$ Speaker. 


\section{Introduction}

When thinking of "relics from the early universe", we usually think of particles. Yet, primordial black holes $(\mathrm{PBH})$ are also hypothetical relics, which can originate from the gravitational collapse of sufficiently large density fluctuations already during the radiation dominated epoch. These fluctuations have characteristic scales much smaller (wavenumber $k \gg \mathrm{Mpc}^{-1}$ ) than the cosmic microwave background (CMB) and large-scale structure (LSS) ones, are typically associated to non-trivial inflationary dynamics or phase transitions, and are largely unconstrained.

A typical PBH mass is a fraction of the mass within the Hubble horizon at the time of its production, $M_{\mathrm{PBH}} \lesssim M_{H}(t) \simeq 5 \times 10^{4} M_{\odot}(t / 1 \mathrm{~s})$. As all $\mathrm{BH}, \mathrm{PBH}$ are subject to "Hawking evaporation" [1], associated to the temperature $T_{\mathrm{H}}=\left(8 \pi G_{N} M\right)^{-1} \simeq\left(10^{13} \mathrm{~g} / M\right) \mathrm{GeV}$ (natural units with $c=\hbar=k_{B}=1$ are adopted, while Newton's constant $G_{N}$ is kept dimensional following particle physics conventions.) The evaporation lifetime (or inverse evaporation rate) rate writes

$$
\tau_{\mathrm{ev}}=\Gamma_{\mathrm{ev}}^{-1}=4 \times 10^{11}\left(\frac{15.35}{\mathscr{F}(M)}\right)\left(\frac{M}{10^{13} \mathrm{~g}}\right)^{3} \mathrm{~s},
$$

where the function $\mathscr{F}(M) \rightarrow 1$ for $M>10^{17} \mathrm{~g}$, saturating at $\mathscr{F} \simeq 15.35$ for $M \lesssim$ few $\times 10^{10} \mathrm{~g}$. For $M>10^{15} \mathrm{~g}, \tau_{\mathrm{ev}}$ exceeds the age of the universe $t_{U}$ : PBH would then constitute a fraction $f_{\mathrm{PBH}}=\Omega_{\mathrm{PBH}} / \Omega_{\mathrm{DM}}$ of the current dark matter $(\mathrm{DM})$, potentially as large as $f_{\mathrm{PBH}}=1$. Nonetheless, bear in mind that lighter PBH may also have a cosmological effect, e.g. in altering primordial nucleosynthesis or playing a role in baryogenesis.

\section{CMB bounds}

The CMB anisotropy pattern is affected by the properties of the medium crossed by the photons between recombination and the present epoch, in particular its ionization fraction $x_{e}$, whose "standard" behaviour vs. redshift $z$ is shown by the black curve in the left panel of Fig. 1. If during this time some electromagnetically (e.m.) interacting particles are injected in the medium, this nonthermal radiation may heat up and especially ionize the gas. It is easy to estimate that alterations to the optical depth experienced by CMB photons make the CMB sensitive even to tiny energy amounts, comparable to a fraction as small as $\mathscr{O}\left(10^{-11}\right)$ of the energy density stored in the DM sector. This effect is ultimately responsible for the well-known CMB sensitivity to annihilating WIMP DM candidates (see [2] and refs. therein for a recent assessment.) But, provided that $T_{\mathrm{H}} \gg 1 \mathrm{eV}$, such modified behaviours in $x_{e}(z)$ are also present in the case of evaporating PBH: some examples are shown with blue/green/red curves in the left panel of Fig. 1. They were computed-thanks to the energy deposition functions calculated in [4] — via a suitable modification of the Boltzmann equations linear anisotropy solver code CLASS (http://class-code.net/). This code also allowed us to derive the modified $\mathrm{CMB}$ angular multipole spectrum: The bounds on evaporating $\mathrm{PBH}$ from the latest Planck CMB data release (retrievable at https://www.cosmos.esa.int/web/planck) with state-of-the-art calculations of the deposited energy are shown with blue shade in the right panel of Fig. 1, where approximate constraints over a larger mass domain (dashed curve), together with previous estimates (orange shaded) and bounds from the gamma-ray diffuse background (red line) are also reported. We clearly see that the CMB offers a complementary probe of "light" PBH with 

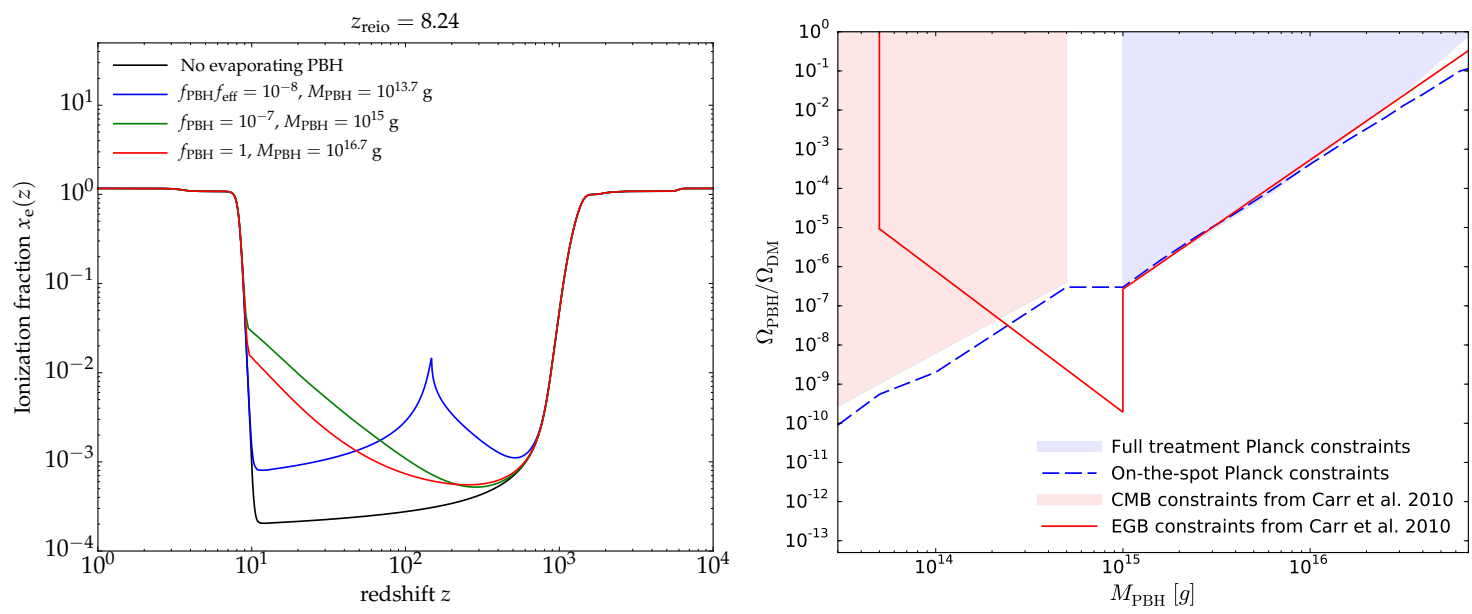

Figure 1: Left: The evolution $x_{e}(z)$ for a standard scenario (black), and for evaporating PBH of different masses and abundances. Right: PBH exclusion limits, above the lines/shaded areas. From Ref. [3].

lifetimes comparable with $t_{U}\left(10^{15} \mathrm{~g} \lesssim M \lesssim 10^{16.5} \mathrm{~g}\right)$, actually leading to the strongest bounds on PBH evaporating over a time a few orders of magnitude shorter than $t_{U}$, for $M$ around $10^{14} \mathrm{~g}$.

For stellar mass BH (including PBH!) evaporation is a negligible phenomenon. Yet, a similar e.m. energy injection is associated to the radiation emitted by the cosmological gas accreting onto $\mathrm{PBH}$, which heats up and gets ionized. Pioneering and extremely stringent bounds obtained a decade ago [5] have been shown to be incorrect in Ref. [6]. In a sufficiently simple, spherical accretion flow model, Ref. [6] derived the very conservative bound $f_{\mathrm{PBH}}<1$ for $M>10-100 M_{\odot}$, the exact value still depending if the ionizing energy of the accreting material is provided by the photons escaping from the hot interior or via collisional effects in the gas. In [7], we have recently argued that the situation considered in [6] is too idealized, and that in a physically plausible framework, stellar mass PBH form accretion disks. Despite lower accretion rates, these configurations are characterized by a significantly larger luminosity than what appropriate for the spherical models of [6]. This results into more stringent bounds, reported in Fig. 2.

Although it was argued in Ref. [8] that the DM may be fully accounted by PBH of tens of solar masses - consistent with the progenitors of the mergers recently detected via gravitational waves by LIGO [9] — by now half a dozen of independent arguments have been raised, indicating that the majority of DM cannot reside in these objects. On the other hand, the possibility of a subleading fraction of DM in the form of stellar-mass PBH accounting for part or even the totality of LIGO merger rate $[10,11]$ is still an open possibility, which makes the investigation of specific signatures particularly important in the forthcoming years.

Another scenario which also requires careful consideration is that PBH may constitute a large (dominant?) fraction of DM, but for masses possibly different from those considered above. Interestingly, CMB can also put bounds on this case. Assume that some fraction $f_{d}$ of DM converts into "dark" radiation - which can be made of new physics degrees of freedom, but also low-energy neutrinos or even gravitational waves $(\mathrm{GW}$ ) - at some characteristic time: this would be the case, for instance, if DM is made by a stable component, plus an unstable relic with lifetime $\tau_{d}$ whose fraction of the initial total is $f_{d}$. Both the smooth and the (linear) fluid perturbations (and thus the 


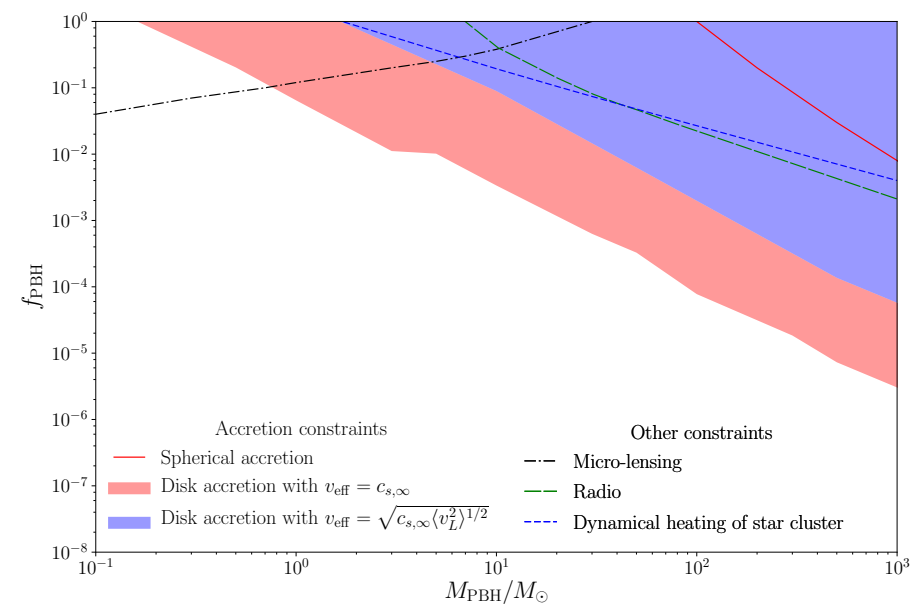

Figure 2: $\quad \mathrm{PBH}$ parameter space excluded by $\mathrm{CMB}$, in the case they accrete via disk formation (for two cases), compared with the spherical accretion (solid, red line) and other bounds (broken lines). From [7].

metric fluctuations) evolve differently in such a Universe, if compared with the $\Lambda \mathrm{CDM}$ model: the CMB is affected (mostly) by the integrated Sachs-Wolfe effect, altering the multipole spectrum at low $\ell$. LSS help in breaking partial degeneracy with curvature and tensor modes. In Ref. [12], we used this effect to prove that a decaying species constituting the whole of DM (i.e. $f_{d}=1$ ) should have a lifetime $\tau_{d} \gtrsim 160$ Gyr: More than one order of magnitude above $t_{U}$, via purely gravitational effects! In [13], we generalized these results to $f_{d}<1$. For $\tau_{d} \gg t_{U}$, it turns out that at leading order the previously obtained bound applies to the combination $\tau_{d} / f$. But for $f_{d}<1$ the parameter space with $\tau_{d} \ll t_{U}$ opens up: For intermediate values of $\tau_{d}$ (say, longer than the recombination time and shorter than the few billion years timescale) the CMB bound is essentially independent of $\tau_{d}$, requiring $f_{d}<3.8 \%$. The fact that the bound in this regime is independent of $\tau_{d}$ means that it applies no matter what is the specific time dependence with which DM converts into dark radiation. In particular, it applies also to mergers of $\mathrm{PBH}$, converting part of their mass into radiation via emission of gravitational waves in the post-recombination epoch. Since in similar-mass BH mergers about 4-5\% of their mass is converted into GW (with long-known expectations [14] matching the first observations [9]), $\mathrm{PBH}$, if constituting a sizable fraction of the $\mathrm{DM}$, cannot have undergone on average more than one merger over the first few billions years after recombination. The lack of significant mass function evolution for any PBH model aiming at explaining the bulk of the DM is an interesting and non-trivial bound. Constraints also apply to the $z=0$ universe as well as the pre-recombination phase, although a detailed study of this scenario is lacking.

Needless to say, the results summarized above cannot make full justice of the rich cosmological phenomenology of PBH. Let us just conclude by mentioning that future CMB missions or $21 \mathrm{~cm}$ tomography can further improve over current sensitivity, as we argued in [13], and that there are additional signatures—not covered here—which remain promising targets of investigation. 


\section{Acknowledgements}

Many thanks to F. Calore, S. Clesse, K. Kohri, and especially J. Lesgourgues and V. Poulin for collaboration of the topics covered here. Support by by the French ANR, Project DMAstro-LHC, ANR-12-BS05-0006 is acknowledged.

\section{References}

[1] S. W. Hawking, "Black hole explosions," Nature 248, 30 (1974).

[2] V. Poulin, P. D. Serpico and J. Lesgourgues, "Dark Matter annihilations in halos and high-redshift sources of reionization of the universe,” JCAP 1512, no. 12, 041 (2015) [arXiv:1508.01370].

[3] V. Poulin, J. Lesgourgues and P. D. Serpico, "Cosmological constraints on exotic injection of electromagnetic energy,” JCAP 1703, no. 03, 043 (2017) [arXiv:1610.10051].

[4] T. R. Slatyer, "Indirect Dark Matter Signatures in the Cosmic Dark Ages II. Ionization, Heating and Photon Production from Arbitrary Energy Injections,” Phys. Rev. D 93, no. 2, 023521 (2016) [arXiv:1506.03812].

[5] M. Ricotti, J. P. Ostriker and K. J. Mack, "Effect of Primordial Black Holes on the Cosmic Microwave Background and Cosmological Parameter Estimates,” Astrophys. J. 680, 829 (2008) [arXiv:0709.0524].

[6] Y. Ali-Haïmoud and M. Kamionkowski, "Cosmic microwave background limits on accreting primordial black holes,” Phys. Rev. D 95, no. 4, 043534 (2017) [arXiv:1612.05644].

[7] V. Poulin, P. D. Serpico, F. Calore, S. Clesse and K. Kohri, "Squeezing spherical cows: CMB bounds on disk-accreting massive Primordial Black Holes,” Phys. Rev. D, in press (2017) [arXiv:1707.04206].

[8] S. Bird et al.,"Did LIGO detect dark matter?,” Phys. Rev. Lett. 116, no. 20, 201301 (2016) [arXiv:1603.00464]

[9] B. P. Abbott et al. [LIGO Scientific and Virgo Collaborations], "Binary Black Hole Mergers in the first Advanced LIGO Observing Run,” Phys. Rev. X 6, no. 4, 041015 (2016) [arXiv:1606.04856].

[10] M. Sasaki, T. Suyama, T. Tanaka and S. Yokoyama, "Primordial Black Hole Scenario for the Gravitational-Wave Event GW150914,” Phys. Rev. Lett. 117, no. 6, 061101 (2016) [arXiv:1603.08338]

[11] S. Clesse and J. García-Bellido, "The clustering of massive Primordial Black Holes as Dark Matter: measuring their mass distribution with Advanced LIGO,” Phys. Dark Univ. 15, 142 (2017) [arXiv:1603.05234].

[12] B. Audren, J. Lesgourgues, G. Mangano, P. D. Serpico and T. Tram, "Strongest model-independent bound on the lifetime of Dark Matter," JCAP 1412, no. 12, 028 (2014) [arXiv:1407.2418].

[13] V. Poulin, P. D. Serpico and J. Lesgourgues, "A fresh look at linear cosmological constraints on a decaying dark matter component,” JCAP 1608, no. 08, 036 (2016) [arXiv:1606.02073].

[14] J. M. Centrella, “The Final Merger of Comparable Mass Binary Black Holes,” AIP Conf. Proc. 873, 70 (2006) [astro-ph/0609172]. 